\title{
Endoscopic ultrasound-guided fine needle aspira- tion diagnosis of extramedullary hematopoiesis in mediastinum
}

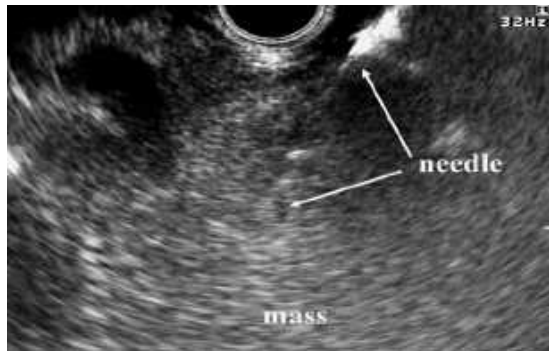

Fig. 1 Endoscopic ultrasonographic (EUS) image demonstrating EUS-guided fine-needle aspiration (EUS-FNA) biopsy of the multinodular, hyperechoic, homogeneous right paravertebral mass; the needle was barely visible during the EUS-FNA because of the hyperechogenicity of the mass.

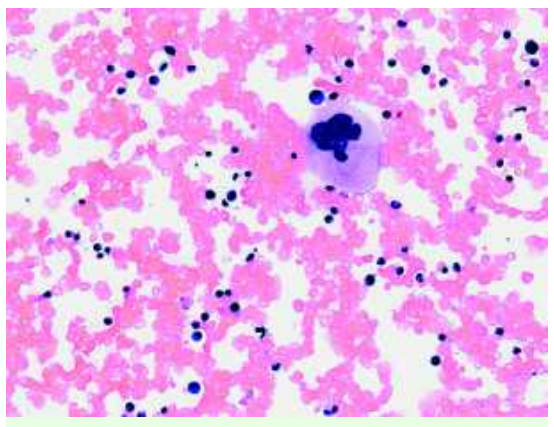

Fig. 2 The smears showed numerous erythroblasts, myelocytes, and granulocytes, and, in the center of the field, a megakaryocyte $(H \& E, \times 100)$.

An 84-year-old woman with thrombotic thrombocytopenic purpura and a past history of bladder carcinoma underwent radiography that disclosed a huge intrathoracic mass, confirmed by CT.

Endoscopic ultrasonography (EUS), performed with an Olympus GF-UCT140AL5 echoendoscope, showed a multinodular, hyperechoic, homogeneous mass in the posterior mediastinum. Fine-needle aspiration (FNA) was performed using a 22-gauge needle (Wilson-Cook, Winston-Salem, North Carolina, USA): the needle was barely visible because of the hyperechogenicity of the mass ( $\bullet$ Fig. 1). Two slides were smeared and stained with hematoxylin-eosin and Giemsa stains by an on-site cytopathologist. The remaining material was preserved by

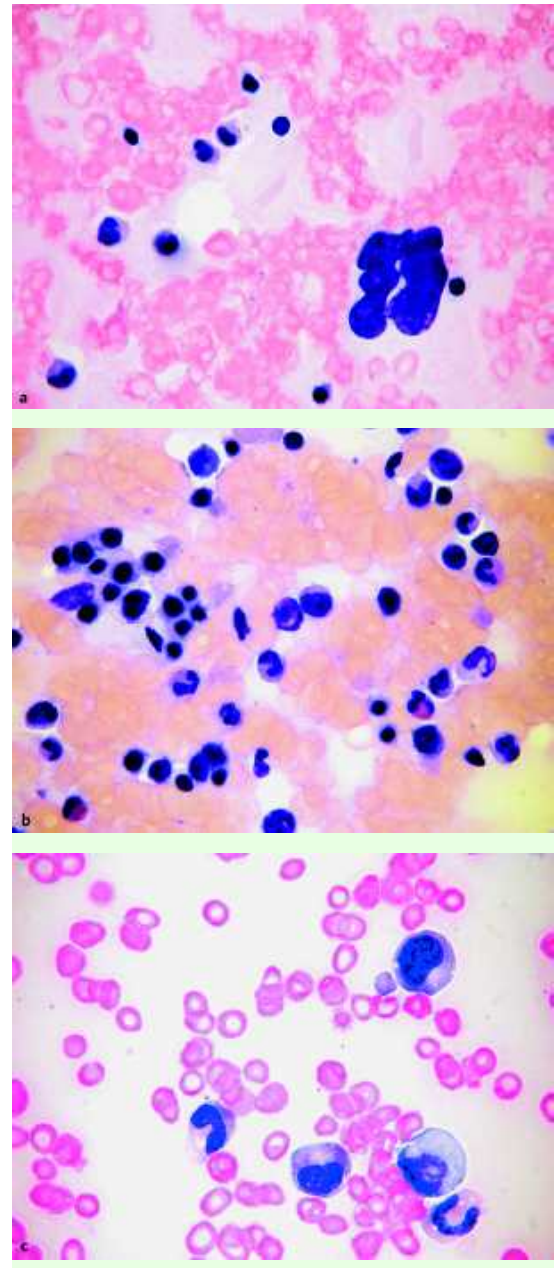

Fig. 3 High-power view of cells and cell precursors from the smear: a megakaryocytes, b erythroblasts (with some myeloid precursors), and c myelocytes ( $\mathrm{H} \& \mathrm{E}, \times 400)$.

95\% ethanol for cell block preparation, subsequently cut and stained with hematoxylin-eosin and Papanicolaou's stain.

The smears, which were moderately cellular, included myeloid precursors, neutrophilic and eosinophilic segmented granulocytes, erythroblasts, and megakaryocytes ( Fig. 2 and Fig. 3).

The sections from the cell block showed trilinear hematopoiesis with prevalence of megakaryocytic and erythroid lineages (๑ Fig. 4).

On the basis of these findings a diagnosis of extramedullary hematopoiesis (EMH)

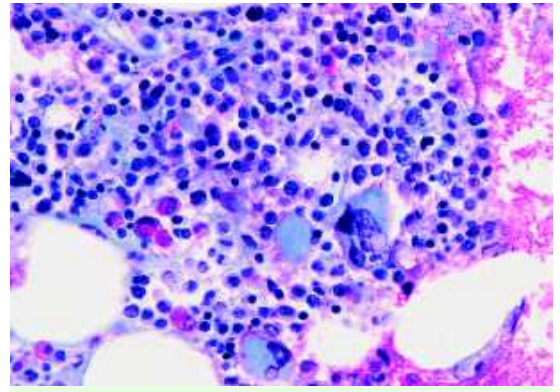

Fig. 4 The tissue fragments from the cell block showed trilinear hematopoiesis with myeloid and erythroid precursors, mature granulocytes and monocytes, megakaryocytes, small lymphocytes, and fat cells (Papanicolaou, $\times 400)$.

was made, ruling out metastatic carcinoma, Hodgkin's lymphoma, and granulocytic sarcoma [1].

$\mathrm{EMH}$, a compensatory response to ineffective intramedullary hematopoiesis, is a rare and benign cause of intrathoracic mass. CT and MRI cannot reliably differentiate benign from malignant disease, so invasive procedures are frequently performed, often requiring hospitalization and general anesthesia. Transthoracic needle biopsy, mediastinoscopy, and thoracoscopy are the standard methods, with minor and major complication rates of up to $10 \%$ and $1.4 \%-2.3 \%$, respectively $[2,3]$. EUS gives an excellent overview of the mediastinum (with the exception of its anterior portion) and EUS-FNA is a minimally invasive technique [4], with a sensitivity, specificity, positive and negative predictive values, and accuracy of $92 \%, 100 \%, 100 \%, 80 \%$, and $94 \%$, respectively [5], allowing more invasive, technically demanding, and expensive procedures to be avoided.

To our knowledge this is the first case of EMH diagnosed by EUS-FNA. Our patient underwent a clinical follow-up, remaining symptom-free after 6 months.

Endoscopy_UCTN_Code_CCL_1AF_2AC 
M. Bosco ${ }^{1}$, P. Carucci ${ }^{2}$, D. Pacchioni ${ }^{1}$, M. Bruno ${ }^{2}$, M. Rizzetto ${ }^{2}$, G. Bussolati ${ }^{1}$, C. De Angelis ${ }^{2}$

1 Department of Biomedical Science and Oncology, University of Turin, Italy

2 Department of Gastrohepatology, University of Turin, Italy

\section{References}

1 Policarpio-Nicolas ML, Bregman SG, Ihsan M, Atkins KA. Mass-forming extramedullary hematopoiesis diagnosed by fine-needle aspiration cytology. Diagn Cytopathol 2006; 34: 807-811

2 Belfiore G, Camera L, Moggio G et al. Middle mediastinum lesions: preliminary experience with CT-guided fine-needle aspiration biopsy with a suprasternal approach. Radiology 1997; 202: 870-873
3 Lemaire A, Nikolic I, Petersen T et al. Nineyear single center experience with cervical mediastinoscopy: complications and false negative rate. Ann Thorac Surg 2006; 82 . 1185 - 1189

4 Buscarini E, De Angelis C, Arcidiacono PG et al. Multicentre retrospective study on endoscopic ultrasound complications. Dig Liver Dis 2006; 38: $762-767$

5 Larsen SS, Krasnik M, Vilmann P et al. Endoscopic ultrasound guided biopsy of mediastinal lesions has a major impact on patient management. Thorax 2002; 57: 98 103
Bibliography

DOI $10.1055 / \mathrm{s}-2008-1077652$

Endoscopy 2009; 41: E6-E7

(C) Georg Thieme Verlag KG Stuttgart · New York . ISSN 0013-726X

\section{Corresponding author}

\section{De Angelis, MD}

S.C. Gastro-Epatologia D.U.

Ospedale Molinette

Corso Bramante 88

Torino

Italy

Fax: +39-01-16335927

eusdeang@hotmail.com 